The 11 year-old woke with left-leg ataxia and sore neck, the morning after being tackled at a football game. He had continued playing to finish the game. Brain stem and cerebellar signs, including left exotropia with nystagmus, impaired adduction of the left eye in convergence, and deviation to the left in compass gait test, were explained by a linear filling defect of the left vertebral artery at C1-2 level consistent with intimal dissection, documented by a 4vessel cerebral arteriogram. CT and brain and cervical spine MRIs were normal. He recovered rapidly and was discharged to take one aspirin daily and avoid contact sports. The 8-year-old also recovered after minor neck trauma followed by recurrent episodes of ataxia, hemiparesis, and visual field defect with left VA thrombus at C-2 and multiple emboli in posterior cerebral arteries. The 7-year-old sustained a traumatic pseudoaneurysm with persistent hemiparesis and ataxia. Clinical data are summarized of 16 cases culled from the literature. (Garg BP, Fishman MA et al. Strokes in children due to

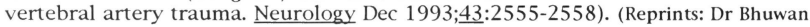
P Garg, Section of Child Neurology, Department of Neurology, Riley Children's Hospital, Rm 1757, 702 Barnhill Drive, Indianapolis, IN 46202).

COMMENT. Fortunately, the prognosis for survival in vertebral artery stroke in children is generally excellent. Only one of 19 patients died.

Two had residual quadriplegia, 9 had mild to moderate residual hemiparesis, ataxia, and/or dysarthria, and 7 (37\%) recovered.

\title{
OUTCOME OF NEONATAL STROKE
}

Evaluations, including MRI, MR angiography, and neuropsychological tests, at 1.5-8.4 years, of 8 infants after neonatal stroke involving the middle cerebral artery are reported from the University of Heidelberg, Mannheim, Germany. Seven had mental and motor retardation and hemiparesis and 4 had epilepsy. Major deficits in cognitive function were found in 4 older children. One patient with normal development showed only a localized temporal lobe lesion on MRI and unremarkable MRA. Children with marked disorders of motor and cognitive development had defects of temporo-parietal lobes, basal ganglia, thalamus and internal capsule on MRI and recanalization of the middle cerebral artery shown on MRA. Those without recanalization had a poorer prognosis. (Koelfen $\mathrm{W}$ et al. Results of parenchymal and angiographic magnetic resonance imaging and neuropsychological testing of children after stroke as neonates. Eur I Pediatr Dec 1993;152:1030-1035). (Respond: Dr W Koelfen,

Department of Pediatrics, Faculty of Clinical Medicine Mannheim, University of Heidelberg,Theodor Kutzer Ufer, D-68167 Mannheim, Germany).

COMMENT. Magnetic resonance angiography (MRA) images intracranial blood vessels without necessity for invasive contrast media. Children with neonatal stroke, seizures, and abnormalities shown on MRA have severe cognitive delays and the least favorable prognosis on long-term follow up.

\section{ASPIRIN-INDUCED NEONATAL INTRACRANIAL HEMORRHAGE}

A term newborn infant with intracranial hemorrhage associated with maternal acetylsalicylic acid ingestion before delivery is reported from the Departments of Pediatrics and Neurology, Eastern Virginia Medical School, Norfolk, VA. Pregnancy was complicated by alcohol abuse. Alka-Seltzer, 6 tablets daily, had been taken for 2 weeks for relief of hangover. The infant's serum salicylate level at 8 hours was $5.4 \mathrm{mg} / \mathrm{dL}$. Hematocrit decreased from 